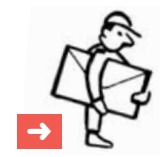

\section{E-Health 2008 - Prozesse definieren und Daten schützen}

Sehr geehrter Herr Giger

Auch nach mehrmaligem Lesen Ihres Editorials [1] kann ich mich des Eindrucks nicht erwehren, dass Ihre Botschaft eigentlich wäre: «Wir haben bald die Health Professional Card (HPC) und suchen dringend nach Anwendungen dafür!»Sie schreiben von Vorleistungen seitens der FMH: HPC, Sicherheit, Autorisierung, elektronische Signatur, sicherer Ausweis, kommunizierte Integrität, medienbruchfreie Unterstützung der Behandlungsprozesse usw. und rufen nach Beschreiben der Prozesse im stationären und ambulanten Sektor, nach kritischer Überprüfung der Schnittstellen usw.

Fazit: Die FMH wird bald ein Tool haben mit diversen Funktionalitäten, das aber in absehbarer Zeit gar nicht zum Einsatz kommen wird. Somit wird diese HPC zum sehr teuren neuen Ausweis. Unsere Warnung, zuerst die Prozesse zu analysieren, zu definieren, allenfalls zu modifizieren und erst dann zu überlegen, welches Tool wir wirklich brauchen, blieb ungehört. - Ich bleibe bei meiner früheren Äusserung: Wir brauchen zuerst eine E-Health-Strategie der FMH, und erst dann kann die HPC skizziert werden. - Ich weiss, der Zug ist abgefahren. Die Delegiertenkammer hat entschieden, der Millionenbetrag ist gesprochen, der Sonderbeitrag wird uns aus dem Sack gezogen.

Trotzdem müssen sich die Verantwortlichen der FMH bewusst werden, dass sie sich damit von der Basis entfernen und ein Akzeptanzproblem vorprogrammiert ist. Diese neue Plastikkarte wird niemanden in Begeisterung ausbrechen lassen ohne eine erste konkrete Anwendung, welche unseren Alltag vereinfacht. Meines Wissens hat die FMH bisher nicht den kleinsten Prozess beschrieben, welcher durch diese HPC vereinfacht würde. Die Kommunikation in diesem Bereich ist katastrophal. Bitte Herr Giger, beschreiben Sie mir eine einzige konkrete Anwendung für die
HPC, die tatsächlich unsere Arbeit vereinfacht und gleichzeitig langfristig die aktuellen Investitionen rechtfertigt.

Heinz Bhend, Aarburg

1 Giger M. E-Health 2008 - Prozesse definieren und Daten schützen. Schweiz Ärztezeitung. 2008; 89(6):203.

\section{Health Professional Card (HPC FMH) mit Anwendungen in Praxis und Berufs- bildung}

Sehr geehrter Herr Bhend

Die HPC wird so rasch und an so vielen Orten angewendet werden können, wie deren Benutzer davon Gebrauch machen wollen. Sie monieren zu Recht die ungenügende elektronische Dokumentation in Praxen und Spitälern. Hier besteht für die Ärzteschaft und Spitalverwaltungen Nachholbedarf. Grund dafür ist wohl die Angst vor Investitionen, deren Nutzen oft anderen zugute kommt. Darauf wird auch im Bericht zur nationalen Strategie «eHealth» verwiesen. Mehraufwand kann abgegolten werden. Der geringe Durchdringungsgrad mit moderner Informations- und Kommunikationstechnologie soll die Schaffung des neuen Ärzteausweises mit der Möglichkeit zur gesicherten elektronischen Kommunikation nicht aufhalten. Die IT-Nutzung wird durch die Vereinfachung der Prozesse, deren sichere Gestaltung, den Einsatz von Kolleginnen und Kollegen sowie die Unterstützung von Schulungen durch die FMH vorangetrieben werden.

Die Gestaltung der Registrierung und des Rollout der HPC beanspruchten mehr Zeit als ursprünglich geplant. Deshalb konnte der Zentralvorstand den Vertrag mit dem Generalunternehmer erst letzte Woche unterschreiben. Die HPC wird Ende 2008/Anfang 2009 allen Mitgliedern der FMH zur Verfügung gestellt werden. Alle Mitglieder werden ein fortgeschrittenes Zertifikat zur sicheren Kommunikation erhalten; qualifizierte Zertifikate können bestellt werden.

Die sichere elektronische Kommunikation erfolgt schon heute. Die HPC soll diese unterstützen. Im Laufe der nächsten Monate werden Pilotprojekte zur Anwendung in grösseren Re- 
gionen aufgebaut. Im Bereich der Weiterbildung wird die Möglichkeit geschaffen, Eingriffe und Untersuchungen digital signiert im Logbuch abzulegen. Im Bereich der Fortbildung soll die Registrierung der Kursbesuche mit der HPC vereinfacht werden.

Die FMH wird in den kommenden Monaten regelmässig über den Stand von Projekten zum Einsatz der HPC und die Umsetzung der natio- nalen Strategie «eHealth» des Bundes orientieren. Wir bitten Sie um Mithilfe bei der praktischen Umsetzung dieser Strategie in den Praxen und laden Sie und weitere Kolleginnen und Kollegen ein, den frisch gewählten Zentralvorstand beim Erfüllen des Auftrags der Ärztekammern 2005 und 2006 zu unterstützen.

Max Giger, Mitglied Zentralvorstand FMH

\section{Briefe an die SÄZ}

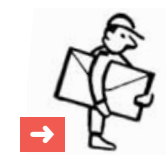

Santésuisse - Wirtschaftlichkeitsverfahren berücksichtigt Facharztgruppen!

In seiner Replik [1] behauptet Dr. Jürg Nadig, santésuisse führe bei der Beurteilung der Wirtschaftlichkeit die statistische Vorselektion mittels ANOVA-Methode unabhängig von der Fachrichtung durch, und fordert eine differenzierte Betrachtung innerhalb der Fachrichtung. Richtig ist, dass santésuisse bereits letzten Sommer damit begonnen hat, die Onkologen und weitere Facharztgruppen für das Statistikjahr 2006 in homogene Untergruppen aufzuteilen. Die Anregungen der Fachgesellschaft für medizinische Onkologie wurden also für die nächstmögliche statistische Umsetzung und Fallbeurteilung berücksichtigt. Entgegen der Behauptung von Dr. Jürg Nadig ist sich santésuisse bewusst, dass die Einteilung nach Facharztgruppen der FMH nicht automatisch zu einer homogenen Verteilung führt.

Die statistischen Berechnungen inklusive ANOVA wurden auf den Untergruppen berechnet. Für die medizinischen Onkologen wurde differenziert nach Fachärzten mit oder ohne intravenöse Chemotherapie in der eigenen Praxis. Bei der Beurteilung der Onkologen mit intravenöser Chemotherapie in der Praxis wurde mittels Regressionsanalyse die Höhe des Anteils der Chemotherapie mitberücksichtigt.

Tatsache ist, dass auch nach Berücksichtigung der Anregungen der Fachgesellschaft einzelne wenige Onkologen bis zu dreimal höhere Fall- kosten als der Durchschnitt der Kollegen des untersuchten Kollektivs aufweisen. Wenn man davon ausgeht, dass sich die Ärzte der Fachgruppe an die empfohlenen Standards halten und die Höhe des Anteils der Chemotherapie bereits berücksichtigt wurde, muss die Wirtschaftlichkeit der Behandlung bei solchen Ausreissern ernsthaft hinterfragt werden. Bei diesen Fällen hat santésuisse im Auftrag der Krankenversicherer die gesetzliche Aufgabe, die betroffenen Ärzte mit der Datenanalyse zu konfrontieren und sofern die massiven Kostenabweichungen nur ungenügend erklärt werden können - ein Rückforderungsverfahren analog demjenigen der Ärzte anderer Facharztgruppen einzuleiten.

Mirjam D'Angelo, lic. rer. pol., Projektleiterin Wirtschaftlichkeitsverfahren, santésuisse

1 Nadig J. Replik auf die Stellungnahme von santésuisse. Schweiz Ärztezeitung. 2008;89(21):907-8.

\section{Kommentar des Autors}

Mit Freuden entnehme ich der Antwort von Frau D'Angelo, dass nun auch santésuisse einsieht, dass ihre bislang angewandte Methode zur Beurteilung der Wirtschaftlichkeit der Medizinischen Onkologen nicht taugt, sondern zu falschen und damit willkürlichen Einschätzungen mit unberechtigten finanziellen Rückforderungen führt. Die Konsequenz daraus müsste sein, dass die eingeleiteten Prozesse sistiert und bereits eingegangene Rückforderungszahlungen den betroffenen Onkologen rückerstattet werden, da sie offensichtlich auf einer falschen Methodik beruhen. Es brauchte offenbar drei Jahre, bis auch santésuisse erkannte, «dass die Einteilung nach Fach- 
arztgruppen der FMH nicht automatisch zu einer homogenen Verteilung führt». Santésuisse hatte es bis anhin eben gerade nicht für nötig erachtet, ihre statistischen Auswertungen auf homogene Untergruppen anzuwenden. Es ist aber fraglich, ob die neue Unterteilung in Onkologen mit oder ohne Chemotherapie tatsächlich reicht, um der Komplexität gerecht zu werden. Das von santésuisse plötzlich aus dem Sack gezauberte, angeblich neue Modell mit Regressionsanalysen hat sie bis heute nicht offengelegt, obwohl vereinbart war, partnerschaftlich mit der Fachgesellschaft Modelle für die Gruppierung der Onkologen zu entwickeln. Ohne das neue Modell zu kennen, kann ich zu seiner Tauglichkeit nicht Stellung nehmen. Noch bevor sich die Ärzte zu dieser neuen statistischen Black Box überhaupt äussern konnten, hat santésuisse in den letzten Tagen bereits wieder mehrere Verfahren gegen praktizierende Onkologen eingeleitet.

Dr. med. Jürg Nadig, Präsident Schweizerische Gesellschaft für Medizinische Onkologie

\section{Verdeckte Rationierung dank Wirtschaftlichkeitsverfahren?}

In seinem Artikel über die Tauglichkeit der ANOVA-Methode von santésuisse veranschaulicht Jürg Nadig [1, 2], warum die Varianzanalyse als statistische Vorselektion zum Herausfiltern auffälliger Onkologen nicht taugt. Woraus der Autor schliesst, dass Fallkosten in der Onkologie im besonderen und Leistungsdaten im allgemeinen normalverteilt sind, ist nicht nachvollziehbar. Die Stellungnahme der santésuisse [3] zum Beitrag geht auf diese unsichere Annahme nicht ein.

Dr. med. Ch. Müller, Thalwil ZH

1 Nadig J. Verdeckte Rationierung dank Wirtschaftlichkeitsverfahren? Schweiz Ärztezeitung. 2008;89(20): 855-60.

2 Nadig J. Replik auf die Stellungnahme von santésuisse. Schweiz Ärztezeitung. 2008;89(21):907-8.

3 D'Angelo M, Rhyn P. Wirtschaftlichkeitsverfahren der santésuisse nach den ANOVA-Methode. Schweiz Ärztezeitung. 2008;89(21):906-8. 